\title{
ARA 290, a Nonerythropoietic Peptide Engineered from Erythropoietin, Improves Metabolic Control and Neuropathic Symptoms in Patients with Type 2 Diabetes
}

\author{
Michael Brines, ${ }^{1,6}$ Ann N Dunne, ${ }^{1}$ Monique van Velzen, ${ }^{2}$ Paolo L Proto, ${ }^{2}$ Claes-Goran Ostenson, ${ }^{3}$ \\ Rita I Kirk, ${ }^{1}$ Ioannis N Petropoulos, ${ }^{4,5}$ Saad Javed, ${ }^{4}$ Rayaz A Malik, ${ }^{4,5}$ Anthony Cerami, ${ }^{1}$ and Albert Dahan ${ }^{2}$ \\ ${ }^{1}$ Araim Pharmaceuticals, Tarrytown, New York, United States of America; ${ }^{2}$ Department of Anesthesiology, Leiden University Medical \\ Center, Leiden, The Netherlands; ${ }^{3}$ Department of Molecular Medicine and Surgery, Karolinska Institutet, Stockholm, Sweden; ${ }^{4}$ Centre \\ for Diabetes and Endocrinology, Institute of Human Development, Manchester Academic Health Science Centre, Manchester, United \\ Kingdom; and ${ }^{5}$ Weill Cornell Medical College in Qatar, Qatar Foundation, Doha, Qatar
}

\begin{abstract}
Although erythropoietin ameliorates experimental type 2 diabetes with neuropathy, serious side effects limit its potential clinical use. ARA 290, a nonhematopoietic peptide designed from the structure of erythropoietin, interacts selectively with the innate repair receptor that mediates tissue protection. ARA 290 has shown efficacy in preclinical and clinical studies of metabolic control and neuropathy. To evaluate the potential activity of ARA 290 in type 2 diabetes and painful neuropathy, subjects were enrolled in this phase 2 study. ARA 290 (4 mg) or placebo were self-administered subcutaneously daily for 28 d and the subjects followed for an additional month without further treatment. No potential safety issues were identified. Subjects receiving ARA 290 exhibited an improvement in hemoglobin $A_{1 c}\left(H b A_{l c}\right)$ and lipid profiles throughout the $56 \mathrm{~d}$ observation period. Neuropathic symptoms as assessed by the PainDetect questionnaire improved significantly in the ARA 290 group. Mean corneal nerve fiber density (CNFD) was reduced significantly compared with normal controls and subjects with a mean CNFD >1 standard deviation from normal showed a significant increase in CNFD compared with no change in the placebo group. These observations suggest that ARA 290 may benefit both metabolic control and neuropathy in subjects with type 2 diabetes and deserves continued clinical evaluation.
\end{abstract}

Online address: http://www.molmed.org

doi: $10.2119 / \mathrm{molmed} .2014 .00215$

\section{INTRODUCTION}

The current worldwide epidemic of type 2 diabetes constitutes one of the major unmet challenges of public health. The chronic diabetic state is characterized by hyperglycemia- and lipidinduced inflammatory processes that damage tissues in a potentially selfamplifying manner (1). While current therapy of diabetes has targeted glucose control, treatment of diabetic complications, for example, neuropathy, aside from striving for good metabolic control has been only symptomatic. An integrated therapeutic approach to diabetes would target both the underlying cause of glucose intolerance as well as activate repair systems to prevent or reverse end organ damage, that is, a disease modifying therapeutic.

Over the past 15 years, preclinical studies have identified the presence of an endogenous protective system that is activated by inflammation, metabolic stress

Address correspondence to Michael Brines, Araim Pharmaceuticals, 580 White Plains Road, Suite 210, Tarrytown, NY 10591. Phone: 914-7627586; Fax: 914-2944173; E-mail: mbrines@araimpharma.com.

Submitted October 27, 2014; Accepted for publication October 27, 2014; Epub (www.molmed.org) ahead of print November 6, 2014.

The Feinstein Institute for Medical Research

North Empowering Imagination. Pioneering Discovery.

and tissue injury (2). The receptor for this response, innate repair receptor (IRR) is a member of the type I cytokine receptor family and is a complex consisting of $\beta$ common receptor (CD131) and erythropoietin (EPO) receptor subunits (3). The principal mediator of this homeostatic response is hypoglycosylated EPO produced in situ by many cells as a stress response. This locally produced EPO has been shown in diverse model systems to antagonize the production and effects of proinflammatory molecules, for example, tumor necrosis factor (TNF) (4), as well as activate healing processes. However, proinflammatory agents also attenuate the local production of EPO. In many disease states, therefore, the balance between tissue damage and healing is tipped toward injury due to inadequate EPO production.

Administration of recombinant human EPO (rhEPO) has been shown to be effec- 
tive in the reduction of inflammation and activation of the healing process in a wide variety of preclinical models as well as in patients. With respect to diabetes, substantial recent work has shown that rhEPO can improve the diabetic state in animal models (5). However, in addition to interacting with the innate repair receptor in a paracrine/autocrine manner, EPO also functions as a circulating hormone via its interaction with the EPO receptor homodimer that mediates hematopoiesis. Therefore, use of rhEPO for treatment of tissue injury is limited by the potential side effects of increased erythrocyte mass and endothelial cell activation, which, in combination with activated platelet production, predisposes to thrombosis $(6,7)$. To circumvent this problem, we have developed engineered proteins and peptides that interact only with the innate repair receptor $(8,9)$.

One promising candidate is ARA 290, which is an 11-amino acid peptide modeled from the three dimensional structure of helix B of the EPO molecule that interacts with the IRR (8). ARA 290 has been evaluated extensively in a wide spectrum of preclinical models, including diet-induced insulin resistance (10), diabetic retinopathy (11), diabetic autonomic neuropathy (12), myocardial infarction (13), chronic heart failure (14), burns (15), traumatic brain injury $(16,17)$ and shock-induced multi-organ failure (18), among others. The results of these studies show that ARA 290 prevents tissue injury, reduces inflammation and activates healing. However, in contrast to rhEPO that has shown side effects in clinical trials, to date ARA 290 has been shown to be safe when formally evaluated in preclinical animal toxicology, normal human volunteers and patients with sarcoidosis (19-21).

Many diseases, including diabetes, have been associated with damage and loss of small unmyelinated or lightly myelinated fibers of the peripheral sensory and autonomic nervous system, termed small fiber neuropathy (SFN). This pathological process is characterized by neuropathic pain, sensory symp- toms and/or autonomic dysfunction (22). Diabetic painful neuropathy has been shown to be mediated via an inflammatory chemokine pathway (23) and strategies to reduce this inflammation, including gene transfer of soluble TNF receptor, have been shown to alleviate pain (24). In preclinical models of neuropathy, ARA 290 has been shown to prevent and improve peripheral neuropathic pain, an action that requires the innate repair receptor (25). In these effects, ARA 290 is not acting as an analgesic agent (26), but rather reduces the underlying inflammation (27) and stimulates nerve fiber regrowth from damaged axons. Recent studies of patients with sarcoidosis and SFN have shown that ARA 290 reduces neuropathic symptoms in conjunction with an increase of small nerve fibers as assessed by changes in the density of corneal nerve fibers (19). Additionally, ARA 290 has been shown to reverse diabetes-induced autonomic nerve degeneration in a murine model (12).

The present study was initiated to determine whether ARA 290 improves metabolic control and neuropathic pain in patients with type 2 diabetes.

\section{MATERIALS AND METHODS}

ARA 290 is a linear 11-amino acid peptide of molecular weight 1257 daltons (21). The material for this study was produced by Bachem (Weil am Rhein, Germany) and prepared in sterile vials allowing for daily subcutaneous self-injection (SC) of $4 \mathrm{mg}$. Placebo consisted of the vehicle $(20 \mathrm{mmol} / \mathrm{L}$ sodium phosphate buffer, pH 6.5, 1\% sucrose and $4 \%$ D-mannitol. The results of pharmacokinetic analyses performed in normal volunteers show that following $4 \mathrm{mg} \mathrm{SC}$, a peak plasma level $\sim 3 \mathrm{ng} / \mathrm{mL}$ $(\sim 2.4 \mathrm{nmol} / \mathrm{L})$ was obtained with a terminal half-life of $\sim 20 \mathrm{~min}$ (21). Preclinical pharmacokinetic-pharmacodynamic data has shown that beneficial effects of ARA 290 occur when plasma concentrations exceed $1 \mathrm{nmol} / \mathrm{L}$ (2).

This double blind, placebo-controlled, investigator-initiated clinical trial is reg- istered in the Netherlands Trial Register (NTR3858). The primary purpose was to determine the safety and efficacy of ARA 290 on metabolic control and neuropathic symptoms in subjects with type 2 diabetes. Primary endpoints were (1) collection of adverse events and laboratory parameters; (2) change in hemoglobin $\mathrm{A}_{1 \mathrm{c}}\left(\mathrm{Hb} \mathrm{A_{1 \textrm {c } }}\right)$ at $\mathrm{d} 28$ and 56 compared with baseline; (3) change in the scores of the Small Fiber Neuropathy Screening List (SFNSL), PainDetect, Neuropathic Pain Symptom Inventory (NPSI), and RAND-36 at d 28 and 56 compared with baseline. Secondary endpoints were change in (1) quantitative sensory testing; (2) corneal nerve fiber density (CNFD); and (3) 6 Minute Walk Test (6MWT) distance at $\mathrm{d} 28$ versus baseline. Primary Inclusion criteria were (1) diagnosis of type 2 diabetes mellitus; (2) spontaneous discomfort level of 6 or greater on a numerical rating scale of the "Pain Now" (0 [none] to 10 [worst]) of the PainDetect questionnaire and SNFSL score of $>22$, or spontaneous discomfort level on Pain Now $<5$ and SFNSL $>44$ at screening and at first dosing visit; (3) discomfort defined as distal pain/discomfort plus one of the following: (a) paresthesia; (b) burning/painful feet worsening at night; or (c) intolerance of sheets or clothes touching the legs or feet; (4) between 18 and 70 years of age; and (5) body mass index (BMI) $<40 \mathrm{~kg} / \mathrm{m}^{2}$. Primary exclusion criteria were (1) vaccination or immunization within the month prior to screening; (2) anti-TNF therapy or other biological antiinflammatory agents administered within the 6 months prior to screening; or (3) use of erythropoiesis stimulating agents within the two months prior to screening or during the trial.

The power analysis was based on data collected from a double-blind trial of the safety and efficacy of ARA 290 in patients with sarcoidosis and symptoms of small fiber neuropathy that received $4 \mathrm{mg}$ ARA 290 SC daily for $28 \mathrm{~d}$ (19). In this study, the mean decrease in the SFNSL score for ARA 290 group at $28 \mathrm{~d}$ was 9.1 points with a standard deviation 
(SD) of 8.5. On the basis of these data, we estimated that to determine a difference between the active and placebo group of a 9-point change in the SFNSL score at a $p$ value of 0.05 with a power of $0.9,40$ patients (20 each arm) be required. On the basis of the prior experience in diabetic populations, we also estimated that up to 10 subjects would potentially withdraw from the study. Therefore, a total of 50 patients ( 25 each patient group) was required to complete this study.

After obtaining informed consent, a total of 61 subjects having diabetes and painful distal extremities were screened: 49 met the inclusion criteria and were enrolled in the trial. One patient discontinued drug administration at d 15 of dosing due to a worsening of borderline renal insufficiency (see Safety section below) and was eliminated from the analysis $(n=48)$. The baseline demographics of subjects are listed in Table 1.

Subjects self-injected active or placebo (0.5 cc total volume) subcutaneously into the anterior thigh using rotating injection sites. Compliance was assessed by a count and visual inspection of vials returned to the pharmacy and checked again via a daily patient diary. Subjects also were maintained on a variety of medications for treatment of diabetes and its complications, including a wide range of drugs to treat neuropathic pain, including opioids, tricyclic antidepressants, nonsteroidal antiinflammatories, serotonin reuptake inhibitors, and/or antiepileptics. These medications were continued throughout the trial.

\section{Endpoint Assessments}

Safety. Clinical chemistry, hematology and vital signs were recorded at enrollment, and at 14, 28 and $56 \mathrm{~d}$. A continuous recording 12-lead electrocardiogram was obtained before and for $10 \mathrm{~min}$ following the first administration of the study drug. Serum for anti-ARA 290 antibodies was obtained at baseline and again at the end of dosing ( $28 \mathrm{~d})$. Subjects were interviewed in person or by telephone every $2 \mathrm{wks}$ and questioned

Table 1. Baseline patient characteristics. ${ }^{a}$

\begin{tabular}{|c|c|c|}
\hline Variable & ARA 290 & Placebo \\
\hline Subjects & 24 & 24 \\
\hline Males/females & $18 / 6$ & $12 / 12$ \\
\hline Weight (kg) & $92.2 \pm 3.8$ & $91.7 \pm 2.7$ \\
\hline Age & $63.0 \pm 1.5$ & $63.8 \pm 1.4$ \\
\hline Height (cm) & $175.3 \pm 1.8$ & $171.0 \pm 1.6$ \\
\hline BMl & $29.9 \pm 1.0$ & $31.4 \pm 0.8$ \\
\hline ARA 290 dose (mcg/kg) & $45.0 \pm 1.9$ & 0 \\
\hline ARA 290 dose $\left(\mathrm{mg} / \mathrm{m}^{2}\right)$ & $1.9 \pm 0.05$ & 0 \\
\hline hs-CRP ${ }^{b}$ & $3.35 \pm 0.86$ & $3.73 \pm 1.17$ \\
\hline $\mathrm{Hb} \mathrm{A}{ }_{\mathrm{lc}}(\% ; \mathrm{mmol} / \mathrm{mol})^{\mathrm{c}}$ & $\begin{array}{r}7.3 \pm 0.4 ; 55.8 \pm 4.4 \\
(5.4 \%, 6.6 \%, 12.3 \%)\end{array}$ & $\begin{array}{c}6.9 \pm 0.2 ; 51.7 \pm 1.9 \\
(5.8 \%, 6.7 \%, 9.3 \%)\end{array}$ \\
\hline Cholesterol (mmol/L) & $4.57 \pm 0.18$ & $4.59 \pm 0.15$ \\
\hline $\mathrm{HDL}(\mathrm{mmol} / \mathrm{L})$ & $1.22 \pm 0.09$ & $1.35 \pm 0.08$ \\
\hline Triglycerides (mmol/L) & $2.35 \pm 0.25$ & $2.30 \pm 0.27$ \\
\hline SFNSL total score & $24.8 \pm 2.5(3,25,52)$ & $21.3 \pm 1.7(7,21,38)$ \\
\hline \multicolumn{3}{|l|}{ RAND-36 } \\
\hline NPSI total score & $17.0 \pm 2.0(0,16.5,33.3)$ & $21.7 \pm 2.0(4.5,22.8,37.5)$ \\
\hline PainDetect total score & $18.0 \pm 1.4(6,20,27)$ & $18.0 \pm 1.3(6,20,28)$ \\
\hline 6MWT (m) & $410.3 \pm 17.4$ & $447.8 \pm 19.6$ \\
\hline $6 \mathrm{MWT}$ predicted $(\mathrm{m})^{\mathrm{c}}$ & $656.0 \pm 13.8$ & $618.4 \pm 13.6$ \\
\hline CNFD (number/mm²) & $24.8 \pm 1.4$ & $24.1 \pm 1.7$ \\
\hline
\end{tabular}

a Values correspond to number or mean \pm SEM. Values within brackets refer to the range as: minimum, median, maximum.

${ }^{b}$ hs-CRP, high-sensitivity C-reactive protein.

${ }^{c}$ For each group, $\mathrm{n}=21$. Only subjects with $\mathrm{A}_{1 \mathrm{c}}$ values at baseline, $\mathrm{d} 28$ and $\mathrm{d} 56$ included $(n=42)$.

${ }^{\circ}$ Calculated from Troosters et al. (35).

about possible adverse events. Adverse events reported spontaneously by subjects outside the above-indicated time points also were documented.

Other endpoints. Neuropathic pain was assessed using the numerical rating scales of PainDetect (28) and the Neuropathic Pain Symptom Inventory (29), two validated and widely used patient reported outcomes. The versions used in this study were validated Dutch translations. Additionally, the SFNSL was used to assess the severity of small fiber neuropathic symptoms. The SFNSL was developed for Dutch-speaking individuals and has been validated in sarcoidosis patients that have symptoms of small fiber neuropathy (30). Finally, the Dutch version of the RAND-36 was utilized to determine health-related quality of life and scoring was carried out as described by Hays, et al. (31).
For missing data of individual questions in the questionnaires, the last data point recorded was carried forward. One placebo patient was missing the baseline PainDetect value and was eliminated from the PainDetect analysis.

Quantitative sensory testing was performed for the face, hand and the foot using a Medoc Advanced Medical Systems device (Ramat Yishai, Israel) in accordance with the German Research Network on Neuropathic Pain protocol (32), but was modified to include only six of the thirteen domains to reduce the amount of time subjects would be involved in testing. These domains were: cold detection threshold (CDT), warm detection threshold (WDT), thermal sensory limen (TSL), paradoxical heat sensation (PHS), allodynia (ALL) and vibration detection threshold (VDT). Normative values used were those of Rolke et al. (33). 
The 6MWT was performed according to the American Thoracic Society guidelines (34). The expected 6-min walk distance was calculated using age, gender and height as per Troosters, et al. (35).

Corneal nerve fiber images were obtained using a Rostock Cornea Module/ Heidelberg Retina Tomograph III and established methodology (36). A minimum of six images from each eye were selected by a single experienced investigator blinded as to treatment, based on the presence of adequate nerve fiber contrast and full field of view. CNFD (number $/ \mathrm{mm}^{2}$ ) was manually quantified by a blinded expert analyst according to our established criteria and evaluation methods (37) For determination of normal corneal nerve fiber density and length, 55 healthy individuals ranging in age from 28 to 73 years (median $=48$ years) were evaluated.

\section{Statistical Analysis}

Data was analyzed using JMP (version 11; SAS, Cary, SC, USA). All variables were considered to be continuous and confirmed to follow a normal distribution before parametric statistical methods used. Statistical testing was performed as delineated in the text. For corneal nerve density, some subjects fell within the normal range. In addition to a comparison of the full subject group, a post hoc analysis was carried out for the group of subjects having a CNFD $>1$ SD less than the normal mean.

\section{RESULTS}

\section{Safety}

No clinically significant changes from baseline values of the hematology or clinical chemistry were observed at any of the time points sampled following ARA 290. Anti-ARA 290 titers at baseline and after $28 \mathrm{~d}$ were negative. The frequency distribution of AEs was similar in both treatment groups: for ARA 290, 54 "mild," 9 "moderate" and 1 "severe" categorizations were recorded, while for placebo, 61 "mild" and 5 "moderate" were noted. Potential relationship to ad- ministration of study drug as assessed by the investigator was: 25 "possibly" and 39 "unlikely" for the ARA 290 group, and 40 "possibly" and 26 "unlikely" for the placebo group.

Four serious adverse events were observed in the ARA 290 treatment arm. Two were judged unlikely to be associated with ARA 290 administration. The remaining two were judged to be possibly related to ARA 290 administration. In one subject on daily furosemide therapy, the dose was increased after wk 2 of the dosing period and the previously borderline renal insufficiency worsened slightly (creatinine rose from $119 \mu \mathrm{mol} / \mathrm{L}$ to $159 \mu \mathrm{mol} / \mathrm{L})$. The safety committee stopped ARA 290 administration. However, furosemide administration continued at the increased dose and renal function did not improve over the follow up period of $6 \mathrm{wks}$. Another subject, male 70 years of age, developed severe cellulitis of the lower extremity requiring hospitalization 2 wks after the last dose of ARA 290 and suffered a fatal myocardial infarction. The safety committee judged the event to be unrelated to ARA 290 treatment.

\section{Metabolic Control}

Forty-two diabetic subjects for whom complete serial $\mathrm{A}_{1 \mathrm{c}}$ determinations were available $(n=42)$ had good baseline glycemic control as evidenced by mean $\mathrm{Hb} \mathrm{A}_{1 \mathrm{c}}$ values of $7.3 \%(55.8 \mathrm{mmol} / \mathrm{mol})$ in the ARA 290 group $(n=21)$ and $6.9 \%$ (52.1 $\mathrm{mmol} / \mathrm{mol}$ ) in the placebo group $(n=21)$. The mean changes from baseline of $\mathrm{Hb} \mathrm{A}_{1 \mathrm{c}}$ at $\mathrm{d} 28$, and $\mathrm{d} 56$ were: $-0.16 \% \pm 0.07(-1.71 \mathrm{mmol} / \mathrm{mol})$ and $-0.21 \% \pm 0.09(-2.24 \mathrm{mmol} / \mathrm{mol})$ for the ARA 290 group and $-0.01 \% \pm 0.07$ $(-0.14 \mathrm{mmol} / \mathrm{mol})$ and $+0.21 \% \pm 0.08$ $(+2.24 \mathrm{mmol} / \mathrm{mol})$ for the placebo groups respectively (Figure $1 ; p=0.002$ repeated measures analysis of variance [ANOVA]). The magnitude of change in $\mathrm{Hb} \mathrm{A}_{1 \mathrm{c}}$ was directly proportional to baseline values in the ARA 290 group, whereas no relationship was observed between baseline and posttreatment values in the placebo group (data not shown).

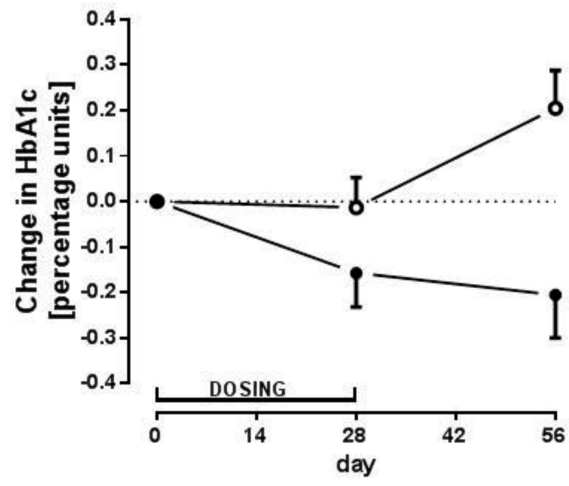

Figure 1. ARA 290 administration is associated with an improvement in $\mathrm{Hb} \mathrm{A}_{1 \mathrm{c}}$ at $\mathrm{d} 28$ and is maintained at least $4 \mathrm{wks}$ following dosing. The $A_{l c}$ percentage decreased significantly from baseline in the group receiving ARA 290 (4 mg SC daily for $28 \mathrm{~d}$; filled circles), whereas the placebo group (open circles) remained unchanged. Follow-up at an additional $28 \mathrm{~d}$ showed the ARA 290 treatment group maintained an improved $A_{1 c}$ profile $(p=$ 0.002; repeated measures ANOVA).

In addition, ARA 290 treatment was associated with significantly reduced ratios of total serum cholesterol to highdensity lipoprotein (HDL)-cholesterol $(p=0.039$; Figure 2A) and serum triglyceride levels ( $p=0.043$; Figure 2C). There was an increase in HDL in the ARA 290 group compared with the placebo group at 28 and $56 \mathrm{~d}$ ( $p=0.066$; Figure $2 \mathrm{~B})$. Similar to the $\mathrm{Hb} \mathrm{A} \mathrm{Ac}_{1 \mathrm{c}}$ results, the change in triglyceride concentration depended upon the baseline value for the ARA 290 group (Figure $3 \mathrm{~A}$ ) but not for the placebo group (Figure 3B).

Diabetic therapeutic regimens varied in the patient population, ranging from no pharmacological treatment to intensive insulin therapy. The majority of subjects experienced no change in medication during the dosing or 1-month follow-up period.

\section{Neuropathic Symptoms}

PainDetect. At baseline, the mean total neuropathic score was identical (ARA 290: $18 \pm 1.37$; placebo $18 \pm 1.29$ ) for the two treatment groups. Distribution of score severity $(\leq 12,13-18$ or $\geq 19)$ was 
A

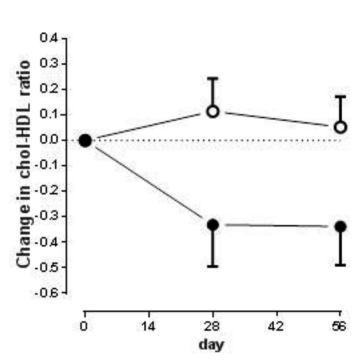

B

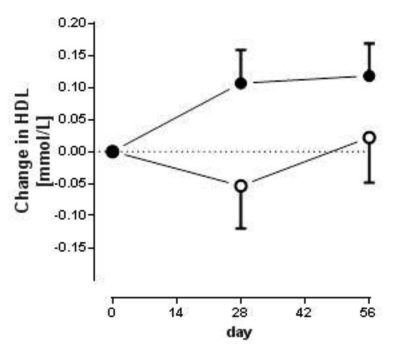

C

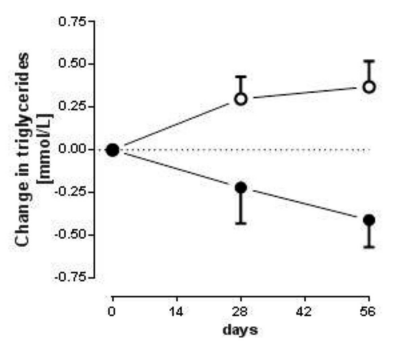

Figure 2. ARA 290 (filled circles) administration exhibited a favorable increase in (A) the cholesterol/high density lipoprotein ratio $(p=0.039)$ and $(B)$ high density lipoprotein $(p=$ 0.066 ), as well as a decrease in (C) triglycerides $(p=0.043)$, in contrast to the placebo group (open circles).

also similar with 6, 12 and 5 subjects in the ARA 290 group and 4, 13 and 6 subjects in the placebo group, respectively. Following dosing, the ARA 290 group improved significantly compared with the placebo group (Figure 4; $p=0.037$ repeated measures ANOVA) with an improvement of 3.3 points in the ARA 290 group versus 1.1 points for placebo. The difference between the groups increased when evaluated at d 56 (28 d following last dose) at which point the mean improvement in the ARA 290 had increased to 4.2 , and the placebo group diminished to 0.74 points. Significant improvements were observed in the "tingling," "thermal pain" and "allodynia" categories ( $p$ values 0.01, 0.04 and 0.04; paired $t$ test), as well as a trend for improvement in the categories of "pressure pain" and "pain attacks" ( $p=0.08$ and $p=0.09)$ in the ARA 290 group, but not in the placebo group. No difference was observed before and after ARA 290 treatment in the "burning" category.

NPSI. The NPSI at baseline indicated mild symptoms (score of $19.4 \pm 1.4$ ). Both groups improved significantly by $\sim 20 \%$ compared with baseline at d 28 (mean decreases of ARA $290=-3.9 \pm 1.9$ and placebo $=-3.7 \pm 1.5 ; p=0.05$ and $p=0.02$ respectively; paired $t$ test). By d 56, neither group was significantly improved compared with baseline (ARA $290=$ $-1.8 \pm 2.3$ and placebo $=-3.1 \pm 2.2$ )

SFNSL. Similar to the NPSI, the baseline total score (out of a maximum of 84) of the SFNSL was in the lower end of the range: 24.8 for the ARA 290 and 21.3 for the placebo group respectively. Neither

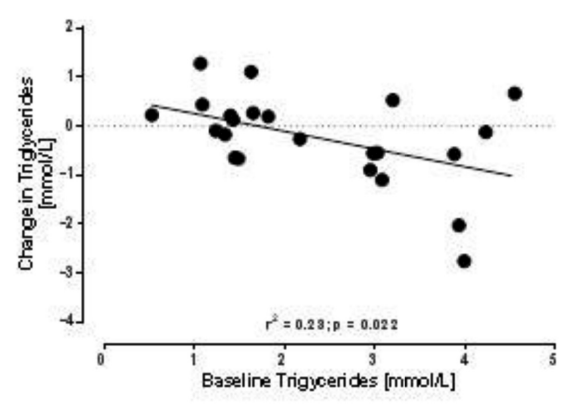

B

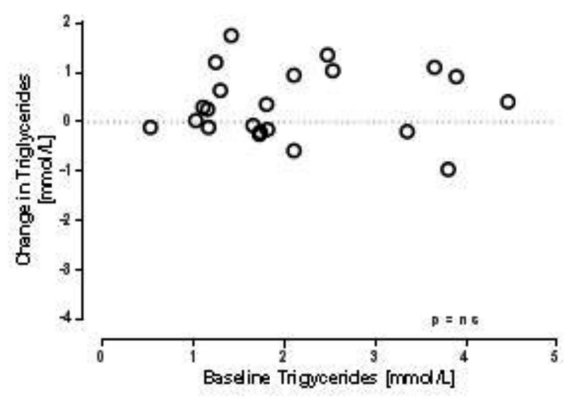

Figure 3. The observed change in triglyceride concentration over the 28-d treatment period was inversely related to baseline level in the ARA 290 group (panel $A ; r^{2}=0.23 ; p=$ 0.022), whereas no relationship was observed for the placebo group (panel $B$ ).

PainDetect

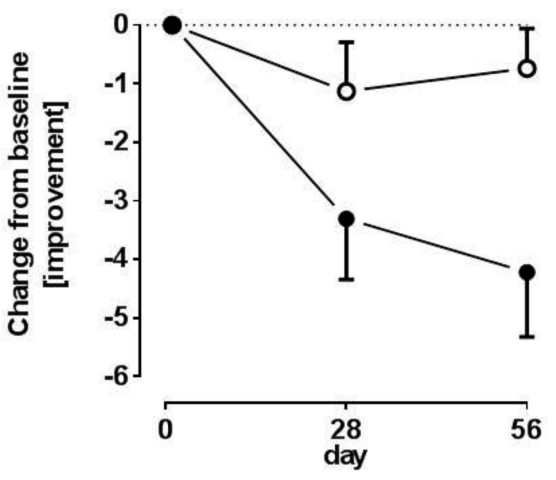

Figure 4. Neuropathic symptoms as assessed by PainDetect are reduced significantly by ARA 290 administration. Time-dependent changes in the total PainDetect score showed a significant improvement in the ARA 290 group (filled circles) compared with placebo (open circles; $p=$ 0.03; repeated measures ANOVA). This corresponds to an $\sim 18 \%$ change and $~ 23 \%$ change from the baseline scores at $d 28$ and d 56 respectively for the ARA 290 group.

the ARA 290 nor placebo treatment group changed significantly from baseline for the SFNSL when assessed at d 28 or d 56.

RAND-36. Baseline scores for each dimension are shown for the two treatment groups with respect to the population norm of patients with chronic disease from the Medical Outcomes Study (31) in Figure 5. The placebo group differed from the reference population only in bodily pain and physical role functioning. In addition to these dimensions, the ARA 290 group differed as well in other dimensions, except for emotional role functioning and mental health. Physical role functioning and vitality (energy/fatigue) was notably reduced in the ARA 290 group. Following 28 d of injections, no changes were observed in the placebo group, whereas the ARA 290 group exhibited significant improvements in the physical role functioning and vitality dimensions ( $p=0.05$ and $p=0.02$ respectively; paired $t$ test). 

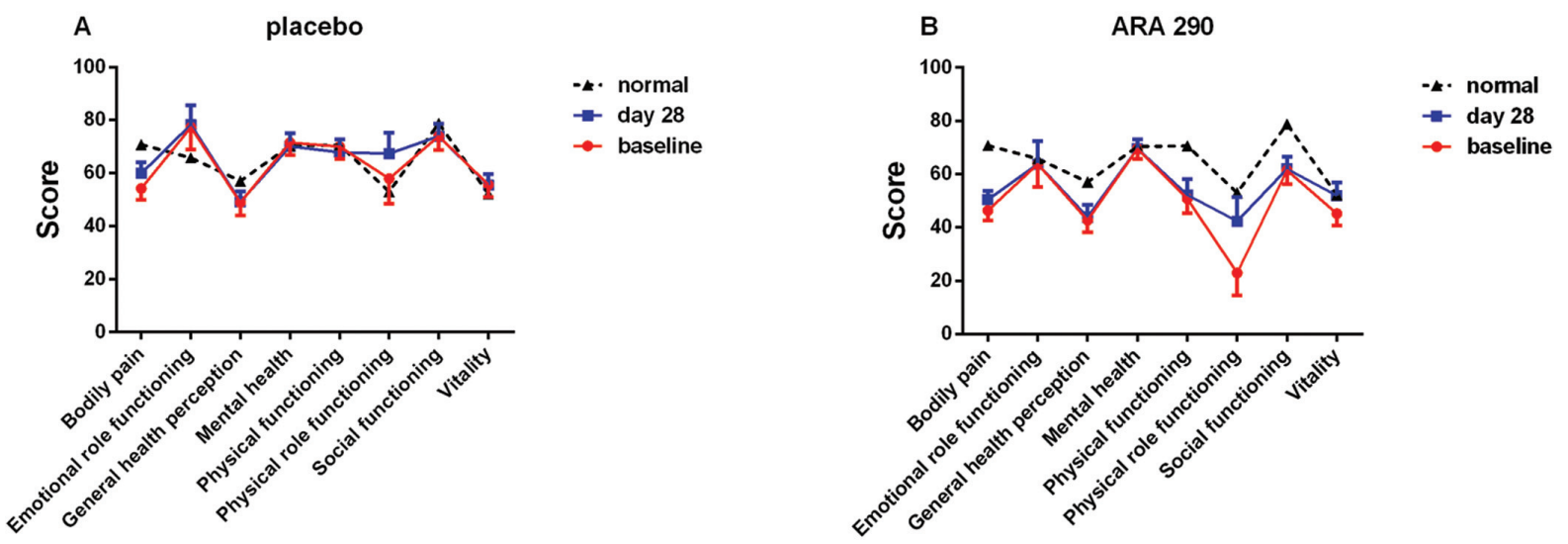

Figure 5. ARA 290 administration benefits several dimensions of the RAND-36. At baseline, the individual dimensions of the RAND-36 in both the ARA 290 (panel B) and placebo (panel A) groups approach the reference population having chronic disease (black dashed line with filled triangles; data from the Medical Outcome Study (31)). The ARA 290 group showed a significant improvement in the physical role functioning and vitality domains. Baseline indicated by filled circles and d 28 by open circles. A higher score indicates a better quality of life. Data points connected as a visual aid. P: pain; RLEP: role limitations emotion problems; GH: general health; E: emotional wellbeing; PF: physical functioning; RLPH: role limitations physical health; SF: social functioning; E/F: energy/fatigue.

6MWT. All subjects performed a $6 \mathrm{MWT}$ at baseline and $\mathrm{d} 28$. The predicted 6-min walk distance calculated from Troosters et al. (35) was a mean of $637 \mathrm{~m}$, whereas the mean 6-min walk distance for the patient cohort was only $429 \mathrm{~m}$. Following dosing, the ARA 290 group increased their walk distance by a mean of $3.5 \%$ of baseline $(p=0.09)$, whereas the placebo group increased walk distance by $1.1 \%$ of baseline ( $p=$ ns [not significant]).

A

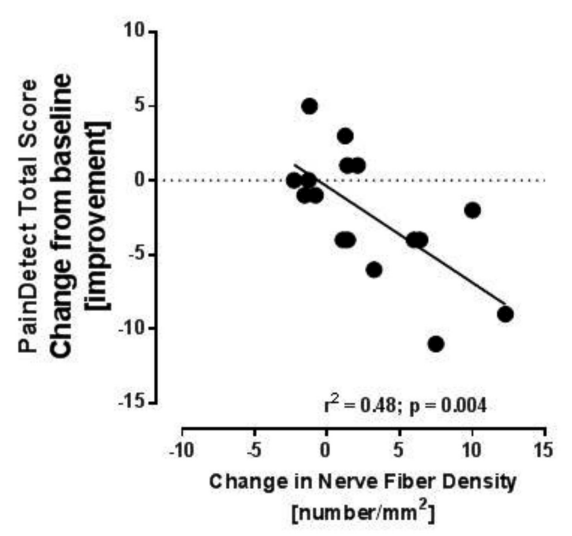

Corneal confocal microscopy. Assessment of corneal nerve fiber density (CNFD) showed that at baseline the mean density was significantly reduced for the diabetes group ( $24.4 \pm 1.1$ fibers $/$ $\mathrm{mm}^{2}$ versus normal control of $37.2 \pm$ 0.9 fibers $\left./ \mathrm{mm}^{2} ; p<0.001\right)$. At d 28 after administering ARA 290, there was a nonsignificant increase of $1.1 \pm 1.1$, compared with the placebo group with a mean increase of $0.1 \pm 1.2$ fibers $/ \mathrm{mm}^{2}$. However, 11 diabetic subjects had a CNFD within

B

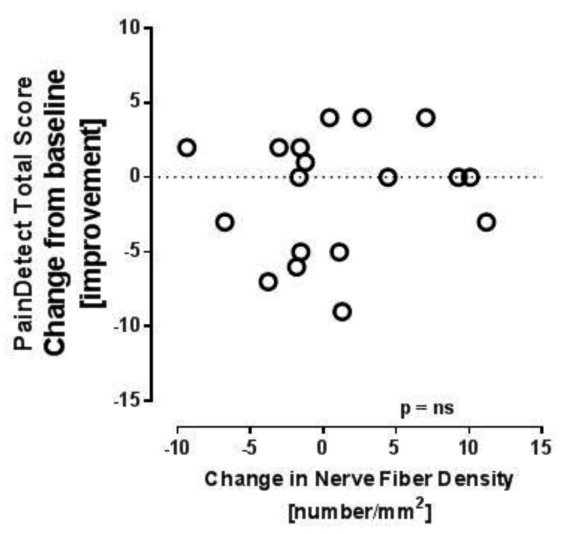

Figure 6. Change in corneal nerve density is related to change in PainDetect total score for the ARA 290 treatment group. In subjects having a baseline CNFD >1 SD from the mean of a normal population, improvement in the PainDetect score was related to the change in CNFD at d 28 in the ARA 290 group (panel A) but not in the placebo group (panel B).
$1 \mathrm{SD}$ of the normal mean and therefore could not be determined to be abnormal. A subgroup analysis performed on subjects having a mean CNFD $>1$ SD from normal was notable for a mean change in of $+2.6 \pm 1.0$ fibers $/ \mathrm{mm}^{2}$ for ARA 290 ( $\mathrm{n}=18 ; p=0.02$; paired $t$ test) versus $+0.7 \pm 1.3$ fibers $/ \mathrm{mm}^{2}$ for placebo $(\mathrm{n}=19$; $p=\mathrm{ns}$ ). Additionally, in this group, a linear relationship existed between the observed change in CNFD and the change in symptoms for the ARA 290 subjects as assessed by PainDetect (Figure 6).

Quantitative sensory testing. Thermal sensitivity testing demonstrated that as a group, at baseline, the subjects exhibited a reduction of function of cold detection threshold of all three regions tested, and reduced function in warm detection threshold in the foot and hand (Figure 7). Additionally, the vibration detection threshold was reduced significantly for the hand and foot. These subjects also suffered from severe allodynia in their feet. There were no significant differences observed after treatment in either patient group.

\section{DISCUSSION}

The results of this study show that ARA 290 (4 mg SC) self-administered for $28 \mathrm{~d}$ by subjects with type 2 diabetes is not 


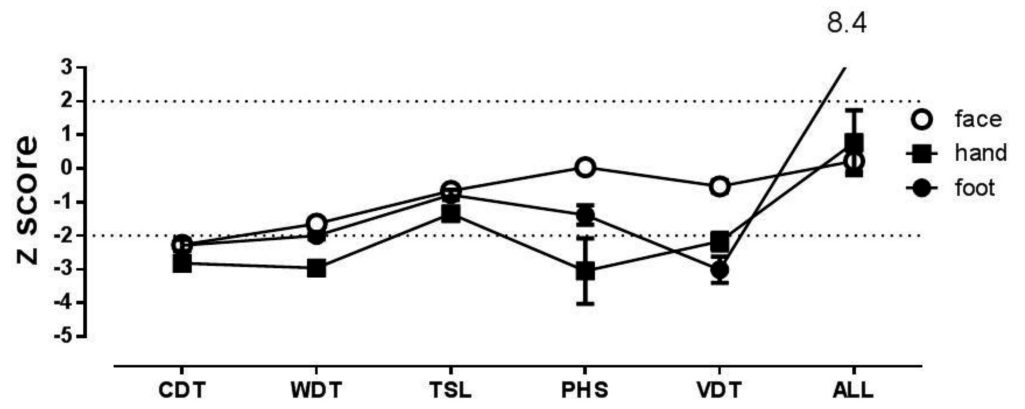

Figure 7. The subjects studied in this trial exhibited both small and large nerve fiber dysfunction. Baseline characteristics of sensory function as assessed using quantitative sensory testing performed using sites on the hand, face, and foot. Thermal thresholds (small fiber function) were especially abnormal, but there also is evidence indicative of large fiber dysfunction as well (vibration and allodynia). The distal extremity exhibited significant allodynia. Negative z scores indicate loss of function and positive score indicate gain of function.

associated with significant ARA 290-related adverse events. Similarly, no clinically significant ARA 290-related alterations of baseline biochemical (electrolytes, calcium, liver, kidney or pancreas) or hematological (erythrocyte, thrombocyte or leukocyte) parameters were observed. In sum, the safety observations support the concept that the IRR, the target of ARA 290 , is induced only by injury, inflammation or metabolic stress and is therefore not generally expressed by normal tissues, thus limiting systemic adverse events.

As predicted by preclinical studies with EPO (5) and ARA 290 (10,38), subjects receiving ARA 290 exhibited significant improvement in glucose control as shown by changes in $\mathrm{Hb} \mathrm{A}_{1 \mathrm{c}}$ concentration. It is notable that the beneficial effect of ARA 290 appears to be sustained for at least the 28-d follow-up period. Although the magnitude of the $A_{1 c}$ change is small, it is consistent with the expectations for antidiabetic agents administered to subjects having excellent baseline control, and is similar to what has been observed in previous clinical trials evaluating antidiabetic agents (39). ARA 290 also benefited the lipid profile, with an improvement in the cholesterol-HDL ratio, in part, as a result of an increase in HDL levels. Additionally, although not performed on fasting serum samples, the triglyceride concentration also improved in parallel to $\mathrm{A}_{1 \mathrm{c}}$. The mechanism(s) of action underlying these changes is currently unclear, but results of preclinical work supports effects of ARA 290 on insulin resistance and muscle mitochondrial biogenesis (10) as well directly on insulin release (38).

The enrollment criteria for this trial required painful neuropathic symptoms of the extremities consistent with small fiber neuropathy. The results of the quantitative sensory testing performed are consistent with a mixed small and large fiber neuropathy. Furthermore, the reduction in corneal nerve fiber density (CNFD) confirms the presence of a structural small fiber neuropathy.

The sensory-related neuropathic symptoms, as assessed by PainDetect, are notable for being quite severe in these well-controlled subjects and are comparable to other trials using this instrument in diabetic subjects (40). Results of a recent study (41) show that this instrument has excellent repeatability and can be used to assess neuropathic symptoms longitudinally. In the current study, ARA 290 significantly improved the total score compared with the placebo group. Changes noted in the scores of individual questions show that symptoms indicative of small fiber function are improved.

Subgroup analysis of those subjects having reduced corneal nerve fibers, and therefore more definitive evidence of small fiber neuropathy (37), show that ARA 290 treatment is associated with an increase in corneal nerve fiber density, analogous to the improvement seen after improved metabolic control (42) and after simultaneous pancreas and kidney transplantation (43). Additionally, the observation that changes in symptoms as assessed by PainDetect are correlated with changes in the CNFD in the ARA 290 treatment group alone, further support a functional relationship between small fiber density and neuropathic symptoms. It is interesting, however, that evaluation of small fiber function as performed by QST (that is, thermal thresholds) did not show significant changes following ARA 290 administration. This is in contrast to a previous trial in sarcoidosis where changes were observed in several temperature-related evaluations (19). Further study is needed to determine the underlying reason for these differences.

Finally, quality of life, as assessed by RAND36, was similar in most dimensions to a large control population with chronic disease in spite of the excellent glycemic control. It is interesting that the changes observed in the ARA 290 group occurred primarily in the vitality (energy/fatigue) dimensions. Fatigue has been observed to be a prevalent and clinically important symptom and major cause of functional disability of subjects with sarcoidosis and SFN (44). It is likely that fatigue is an important component of the symptom complex of these diabetic subjects, which was not formally assessed in this trial.

In summary, ARA 290 shows significant potential for the treatment of diabetic small fiber neuropathy. The two major limitations of this trial are that the treatment duration was only for $28 \mathrm{~d}$ and the subjects were under generally excellent metabolic control. Longer duration of therapy provided to poorly controlled subjects could result in larger changes in $\mathrm{Hb} \mathrm{A}_{1 \mathrm{c}}$ as well as more substantial changes in neuropathic symptoms and objective assessment of small 
nerve fiber density and function. The observed excellent safety profile, along with the positive results presented herein, support further evaluation of ARA 290 as a disease modifying agent in subjects with painful diabetic neuropathy.

\section{ACKNOWLEDGMENTS}

This work was supported in part by a grant from the Dutch government to the Netherlands Institute for Regenerative Medicine (NIRM, grant no. FES0908, the Swedish Research Council, ALF, and the Swedish Diabetes Association). The authors thank Ferdinand C Breedveld and Geertrui Betgen for invaluable assistance, as well as the subjects and their families for agreeing to participate in this trial.

\section{DISCLOSURES}

M Brines, AN Dunne, and A Cerami are officers of Araim Pharmaceuticals and own stock and/or stock options.

\section{AUTHOR CONTRIBUTIONS}

$\mathrm{M}$ Brines designed the trial, analyzed data and wrote the manuscript. AN Dunne designed the trial, oversaw subject recruitment and trial management and wrote the manuscript. $M$ van Velzen conducted clinical aspects of the trial, researched data and participated in the data analyses. PL Proto conducted clinical aspects of the trial, researched data and participated in the data analyses. C-G Ostenson participated in interpretation of the data. RI Kirk contributed to preparation and management of the clinical trial. IN Petropoulos and S Javed undertook quantification of corneal nerve morphology. RA Malik oversaw the CCM quantification and interpretation. A Cerami designed the trial, participated in data analysis and wrote the paper. A Dahan was the Principal Investigator and was involved in all aspects of the trial. All authors contributed to discussion, reviewed and edited the manuscript, as well as approved its final form.

\section{REFERENCES}

1. Navarro JF, Mora C. (2005) Role of inflammation in diabetic complications. Nephrol. Dial. Transplant. 20:2601-4.

2. Brines M, Cerami A. (2012) The receptor that tames the innate immune response. Mol. Med. 18:486-96.

3. Brines M, et al. (2004) Erythropoietin mediates tissue protection through an erythropoietin and common beta-subunit heteroreceptor. Proc. Natl. Acad. Sci. U. S. A. 101:14907-12.

4. Brines M, Cerami A. (2008) Erythropoietin-mediated tissue protection: reducing collateral damage from the primary injury response. J. Intern. Med. 264:405-32.

5. Woo M, Hawkins M. (2014) Beyond erythropoiesis: emerging metabolic roles of erythropoietin. Diabetes 63:2229-2231.

6. Corwin HL, et al. (2007) Efficacy and safety of epoetin alfa in critically ill patients. N. Engl. J. Med. 357:965-76.

7. Robertson CS, et al. (2014) Effect of erythropoietin and transfusion threshold on neurological recovery after traumatic brain injury: a randomized clinical trial. Jama 312:36-47.

8. Brines M, et al. (2008) Nonerythropoietic, tissueprotective peptides derived from the tertiary structure of erythropoietin. Proc. Natl. Acad. Sci. U. S. A. 105:10925-30.

9. Leist M, et al. (2004) Derivatives of erythropoietin that are tissue protective but not erythropoietic. Science. 305:239-242.

10. Collino M, et al. (2014) A non-erythropoietic peptide derivative of erythropoietin decreases susceptibility to diet-induced insulin resistance in mice. Br. J. Pharmacol. 171:5802-15.

11. McVicar CM, et al. (2011) Intervention with an erythropoietin-derived peptide protects against neuroglial and vascular degeneration during diabetic retinopathy. Diabetes. 60:2995-3005.

12. Schmidt RE, et al. (2011) Effect of insulin and an erythropoietin-derived peptide (ARA290) on established neuritic dystrophy and neuronopathy in Akita (Ins2 Akita) diabetic mouse sympathetic ganglia. Exp. Neurol. 232:126-35.

13. Ahmet I, et al. (2011) A small nonerythropoietic helix B surface peptide based upon erythropoietin structure is cardioprotective against ischemic myocardial damage. Mol. Med. 17:194-200.

14. Ahmet I, et al. (2013) Chronic administration of small nonerythropoietic peptide sequence of erythropoietin effectively ameliorates the progression of postmyocardial infarction-dilated cardiomyopathy. J. Pharmacol. Exp. Ther. 345:446-56.

15. Bohr S, et al. (2013) Alternative erythropoietinmediated signaling prevents secondary microvascular thrombosis and inflammation within cutaneous burns. Proc. Natl. Acad. Sci. U. S. A. 110:3513-8.

16. Robertson CS, et al. (2013) Treatment of mild traumatic brain injury with an erythropoietinmimetic peptide. J. Neurotrauma. 30:765-74

17. Robertson CS, et al. (2012) Neuroprotection with an erythropoietin mimetic peptide (pHBSP) in a model of mild traumatic brain injury complicated by hemorrhagic shock. J. Neurotrauma. 29:1156-66.

18. Patel NS, et al. (2011) A nonerythropoietic peptide that mimics the 3D structure of erythropoietin reduces organ injury/dysfunction and inflammation in experimental hemorrhagic shock. Mol. Med. 17:883-92.

19. Dahan A, et al. (2013) ARA 290 improves symptoms in patients with sarcoidosis-associated small nerve fiber loss and increases corneal nerve fiber density. Mol. Med. 19:334-45.

20. Heij L, et al. (2012) Safety and efficacy of ARA 290 in sarcoidosis patients with symptoms of small fiber neuropathy: a randomized, doubleblind pilot study. Mol. Med. 18:1430-6.

21. Niesters M, et al. (2013) The erythropoietinanalogue ARA 290 for treatment of sarcoidosisinduced chronic neuropathic pain. Exp. Opin. Orphan Drugs 1:77-87.

22. Hoeijmakers JG, Faber CG, Lauria G, Merkies IS, Waxman SG. (2012) Small-fibre neuropathiesadvances in diagnosis, pathophysiology and management. Nat. Rev. Neurol. 8:369-79.

23. Menichella DM, et al. (2014) CXCR4 chemokine receptor signaling mediates pain in diabetic neuropathy. Mol. Pain. 10:42.

24. Ortmann KL, Chattopadhyay M. (2014) Decrease in neuroimmune activation by HSV-mediated gene transfer of TNF $\alpha$ soluble receptor alleviates pain in rats with diabetic neuropathy. Brain Behav. Immun. 41:144-51.

25. Swartjes M, et al. (2011) ARA290, a peptide derived from the tertiary structure of erythropoietin, produces long-term relief of neuropathic pain: an experimental study in rats and $\beta$-common receptor knockout mice. Anesthesiology. 115:1084-92.

26. Swartjes M, et al. (2013) Ketamine does not produce relief of neuropathic pain in mice lacking the $\beta$-common receptor (CD131). PLoS One. 8:e71326.

27. Swartjes M, et al. (2014) ARA 290, a peptide derived from the tertiary structure of erythropoietin, produces long-term relief of neuropathic pain coupled with suppression of the spinal microglia response. Mol. Pain. 10:13.

28. Freynhagen R, Baron R, Gockel U, Tolle TR. (2006) painDETECT: a new screening questionnaire to identify neuropathic components in patients with back pain. Curr. Med. Res. Opin. 22:1911-20.

29. Bouhassira D, et al. (2004) Development and validation of the Neuropathic Pain Symptom Inventory. Pain. 108:248-57.

30. Hoitsma E, De Vries J, Drent M. (2011) The small fiber neuropathy screening list: Construction and cross-validation in sarcoidosis. Respir. Med. 105:95-100.

31. Hays RD, Sherbourne CD, Mazel RM. (1993) The RAND 36-Item Health Survey 1.0. Health Econ. 2:217-27.

32. Rolke R, et al. (2006) Quantitative sensory testing: a comprehensive protocol for clinical trials. Eur. J. Pain. 10:77-88. 
33. Rolke R, et al. (2006) Quantitative sensory testing in the German Research Network on Neuropathic Pain (DFNS): standardized protocol and reference values. Pain. 123:231-43.

34. (2002) ATS statement: guidelines for the sixminute walk test. Am. J. Respir. Crit. Care Med. 166:111-7.

35. Troosters T, Gosselink R, Decramer M. (1999) Six minute walking distance in healthy elderly subjects. Eur. Respir. J. 14:270-4.

36. Tavakoli M, Malik RA. (2011) Corneal confocal microscopy: a novel non-invasive technique to quantify small fibre pathology in peripheral neuropathies. J. Vis. Exp. doi: 10.3791/2194.

37. Petropoulos IN, et al. (2014) Rapid automated diagnosis of diabetic peripheral neuropathy with in vivo corneal confocal microscopy. Invest. Ophthalmol. Vis. Sci. 55:2071-8.

38. Muller C, et al. (2013) The nonhematopoietic erythropoietin analogue ARA 290 improves glucose tolerance by stimulating insulin secretion in spontaneously type 2 diabetic Goto-Kakizaki rats. Diabetologia. 56(Suppl1):S268.

39. Dejager S, Schweizer A, Foley JE. (2012) Evidence to support the use of vildagliptin monotherapy in the treatment of type 2 diabetes mellitus. Vasc. Health Risk Manag. 8:339-48.

40. Baron R, Tolle TR, Gockel U, Brosz M, Freynhagen R. (2009) A cross-sectional cohort survey in 2100 patients with painful diabetic neuropathy and postherpetic neuralgia: Differences in demographic data and sensory symptoms. Pain. 146:34-40.

41. Baron R, et al. (2013) Test and re-test of the painDETECT-Questionnaire. Presented at: Proceedings of the 4th International Congress on Neuropathic Pain; 2013 May 23-26; Toronto, Canada.

42. Tavakoli M, et al. (2011) Corneal confocal microscopy detects improvement in corneal nerve morphology with an improvement in risk factors for diabetic neuropathy. Diabet. Med. 28:1261-7.

43. Tavakoli M, et al. (2013) Corneal confocal microscopy detects early nerve regeneration in diabetic neuropathy after simultaneous pancreas and kidney transplantation. Diabetes. 62:254-60.

44. Drent M, Lower EE, De Vries J. (2012) Sarcoidosisassociated fatigue. Eur. Respir. J. 40:255-63. 